reconfiguration of hospital services can provide a powerful means of improving the quality of patient care. The issue as to whether GPs should be more involved with IBD care must be addressed and the patient's attitudes and perceptions of the GP and potential role, should be a focus when restructuring healthcare services.

Methods 24 in depth qualitative interviews with 24 IBD patients were conducted as part of a larger study to develop a model of follow-up care for patients with IBD. 18 patients had Crohns Disease, 6 ulcerative colitis, age range $27-72$ years, disease duration range was $2-40$ yrs. Interviews were 40 to 60 min duration. Patients were asked directly about the role of their GP in their diagnosis and ongoing care of their IBD. Thematic analysis of interviews was undertaken using NVivo 9.0.

Results Many patients experienced years of symptoms prior to diagnosis, in one case diagnosis took 10 years. This misdiagnosis/ mistreatment led to a loss of confidence in the GP for future care. This impacted upon the question if they seek help from their GP at times of flares or IBD related problems. All patients reported no they do not seek help from their GP. This loss of confidence at diagnosis promoted views that their GP did not have the level of knowledge nor expertise to manage ongoing care. This was the same for patients with complex and quiescent disease. Patients would be happy to increase the level of input from their GP but this must be under the direction of the IBD team. Patients do not wish to be discharged completely from the IBD team and would accept reduced intervention and face to face contact with their IBD team, such as telephone and virtual clinics, in order to remain under the care of the IBD team.

Conclusion GPs are the sole carers for a minority of IBD patients. While patients are willing to accept greater involvement from their GP there is general lack of confidence in their expertise to do this effectively. This is reflected by patients expressing a wish to remain under the direct care of the IBD team within secondary care. It is clear from the data that there may be an educational developmental need for the GPs regarding IBD but there is potential for reconfiguration of IBD services in which the GP plays a greater role.

Competing interests None declared.

\section{PTU-096 THE USE OF COMMERCIAL INTERFERON- $\gamma$ RELEASE ASSAYS TO SCREEN FOR MYCOBACTERIAL INFECTION IN INFLAMMATORY BOWEL DISEASE PATIENTS INITIATING ANTI-TNF AGENTS}

doi:10.1136/gutjnl-2012-302514c.96

${ }^{1} \mathrm{~K}$ Greveson, ${ }^{1} \mathrm{C}$ Murray, ${ }^{1} \mathrm{~J}$ Goodhand, ${ }^{2} \mathrm{C}$ Cropley, ${ }^{3} \mathrm{~S}$ Murthy, ${ }^{1} \mathrm{M}$ Hamilton, ${ }^{3} \mathrm{M}$ Lipman. ${ }^{1}$ Centre for Gastroenterology, Royal Free Hospital, London, UK; ${ }^{2}$ Infectious Diseases, Royal Free Hospital, London, UK; ${ }^{3}$ Respiratory Medicine, Royal Free Hospital, London, UK

Introduction Two commercial Interferon Gamma release assays (IGRA) are approved in the UK by NICE and US FDA to detect $M$ tuberculosis (Mtb) infection. The T-Spot.TB (TSTB) and Quanitferon Gold In-tube (OFGIT) use different test platforms, with the potential for slightly different results when used in clinical practice. Since 2008, we have used a standard IGRA-based assessment for Mtb infection in inflammatory bowel disease (IBD) patients. Initially this involved TSTB but in December 2010, switched to OFGIT. Here we review the performance of these assays within our protocol.

Methods We prospectively screened 148 adult IBD patients considered for anti-TNf $\alpha$ agents with symptom review, chest radiograph and IGRA. Between October 2008 and November 2010, 91 patients were tested with TSTB, and between December 2010 and November 2011, 57 with OFGIT. IGRA results were reported as positive, negative or indeterminate. Positive and indeterminate results were referred to $\mathrm{TB}$ services.

Results All subjects had normal chest radiographs and a negative clinical assessment. Overall 82\% (121/148) subjects tested were BCG vaccinated and 13\% (19/148) had risk factors for Mtb. 74\% (109/ 148) were taking immunomodulators. $98 \%(89 / 91)$ patients in the TSTB group had an unequivocal result [1\% (1/91) positive: $97 \%$ (88/ 91) negative], and $1 \%(1 / 91)$ had an indeterminate result, compared with $86 \%$ unequivocal [0\% positive/86\% negative] and $14 \%(8 / 57)$ indeterminate in the OFGIT group, respectively $(p=0.002) .12 \%$ indeterminate results occurred in subjects taking immunosuppression. $85 \%(126 / 148)$ of the anti-TNF $\alpha$ naive group have subsequently received treatment with either infliximab or adalimumab. None have gone on to develop tuberculosis. Median follow-up from start of therapy in the TSTB group is 21 months (IOR 15-26 months); and that of the OFGIT group 7 months (IOR 4-9).

Conclusion We find little evidence for Mtb infection within our IBD population. To date none have developed active TB after starting anti-TNF $\alpha$ therapy. Given the reasonable median follow-up (21 months for TSTB and 7 months for QFGIT) compared to the reported time of onset of active TB following anti-TNF $\alpha$ agents ( $<3$ months), our data provide some reassurance that we have not missed LTBI using our assessment. There appears to be a higher frequency of indeterminate results using OFGIT. As this is found almost exclusively in those on immunosuppressive agents, we suggest that IBD services need to understand the characteristics of the IGRA used within their population and the implications of this for management.

Competing interests None declared.

\section{PTU-097 MEDICAL RESOURCE UTILISATION AND COSTS IN PATIENTS WITH ULCERATIVE COLITIS IN THE UK: A CHART REVIEW ANALYSIS}

doi:10.1136/gutjnl-2012-302514c.97

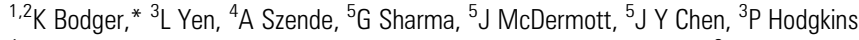
${ }^{1}$ Digestive Diseases Centre, Aintree University Hospital, Liverpool, UK; ${ }^{2}$ Department of Gastroenterology, Institute of Translational Medicine, University of Liverpool, Liverpool, UK; ${ }^{3}$ Shire Development LLC, Wayne, USA; ${ }^{4}$ Covance Market Access Services, Leeds, UK; ${ }^{5}$ Covance Market Access Services, Gaithersburg, USA
\end{abstract}

Introduction Limited evidence is available on the economic burden of ulcerative colitis (UC) in the UK, particularly in relation to the impact of relapse frequency on direct medical costs. The objective of this study was to quantify annual disease-related and all-cause (total) medical resource utilisation (MRU) and associated direct medical costs.

Methods A retrospective chart review of patients with mild-tomoderate UC diagnosed at least 1 year prior to the study was performed. From 33 general practitioner (GP) and 34 gastroenterologist sites in UK, charts of the last three UC patients meeting the study's inclusion criteria were reviewed. 2009-2010 National Health Service reference costs were assigned to the recorded medical resources. Descriptive statistics were calculated for disease-related and all-cause MRU and costs by number of relapses. Logistic regression was used to estimate MRU and costs while accounting for relapse status, patient demographics, site type, and treatment setting.

Results Study population: N=201 patients; mean age: 39.9 years; $44 \%$ female; mean disease duration: 7.4 years. UC-related costs of each MRU category increased with the number of relapses. Comparing those without relapse to patients with $>2$ relapses, mean annual UC-related costs were $£ 14$ vs $£ 2556$ for hospitalisations; £239 vs £2221 for visits; £21 vs £1303 for procedures; £16 vs $£ 188$ for diagnostics. Age, gender, and site of data reporting (GP vs gastroenterologist) were not related to MRU or costs. 
Abstract PTU-097 Table 1 Average annual, UC-related and all-cause per patient Cost (£), by relapse status

\begin{tabular}{|c|c|c|c|c|c|c|}
\hline & 0 relapses $(n=70)$ & 1 relapse $(n=77)$ & 2 relapses $(n=34)$ & $>2$ relapses $(n=20)$ & All $(N=201)$ & p Value \\
\hline UC-related medical costs & 269.5 & 1174.8 & 1768.6 & 5034.7 & 1344.1 & $<0.0001$ \\
\hline Diagnostic tests & 16.5 & 88.4 & 120.1 & 187.7 & 78.6 & $<0.001$ \\
\hline GP visits & 101.8 & 258.9 & 260.1 & 409.2 & 219.3 & $<0.001$ \\
\hline Gastroenterologist visits & 107 & 264.7 & 330.4 & 473.2 & 241.6 & $<0.001$ \\
\hline Nurse visits & 8.9 & 23.3 & 37.9 & 35.1 & 21.9 & 0.0132 \\
\hline ER visits & 21 & 133.2 & 65.4 & 1303.1 & 199 & 0.0039 \\
\hline Outpatient procedures & 0 & 6.6 & 22.6 & 70.4 & 13.4 & $<0.001$ \\
\hline Hospitalisations & 14.3 & 399.8 & 932.2 & 2556 & 570.1 & 0.0006 \\
\hline Total all-cause cost & 1208.5 & 1842.6 & 2672.1 & 6075.2 & 2183.2 & $<0.001$ \\
\hline
\end{tabular}

Conclusion Patients with mild-to-moderate UC have considerable care costs which increase significantly with the number of relapses. These findings support the importance of maintenance therapies in UC that aim to reduce relapse. Quantifying the relationship between relapse rate and costs will inform future health economic studies.

Competing interests $\mathrm{K}$ Bodger: Consultant for: Dr Bodger received funding from Covance Market Access Services, L Yen: Shareholder with: MsYen is a Shire Development LLC, shareholder, Employee of: MsYen is a Shire Development LLC, employee, A Szende: Grant/Research Support from: Dr Szende is a Covance Market Access Services (CMA) employee CMA received funding from Shire, G Sharma: Grant/Research Support from: Dr Sharma is a Covance Market Access Services (CMA) employee CMA received funding from Shire, J McDermott Grant/Research Support from: Mr McDermott is a Covance Market Access Services (CMA) employee CMA received funding from Shire, $\mathrm{J}$ Chen: Grant/Research Support from: MsChen is a Covance Market Access Services (CMA) employee CMA received funding from Shire, P Hodgkins Shareholder with: Dr Hodgkins is a Shire Development LLC, shareholder, Employee of: Dr Hodgkins is a Shire Development LLC, employee.

\section{PTU-098 THE UK INFLAMMATORY BOWEL DISEASE AUDIT: KEY FINDINGS FROM THE INPATIENT EXPERIENCE QUESTIONNAIRE}

doi:10.1136/gutjnl-2012-302514c.98

${ }^{1,2}{ }^{K}$ Bodger, ${ }^{*}{ }^{3}$ Arnott, ${ }^{4} \mathrm{C}$ Down, ${ }^{5} \mathrm{R}$ Driscoll, ${ }^{4} \mathrm{~A}$ Protheroe, ${ }^{4} \mathrm{M}$ Roughton. ${ }^{1}$ Department of Gastroenterology, Institute of Translational Medicine, University of Liverpool, Liverpool, UK; ${ }^{2}$ Digestive Diseases Centre, Aintree University Hospital, Liverpool, UK; ${ }^{3}$ Gastroenterology Department, Western General Hospital, Edinburgh, UK; ${ }^{4}$ Clinical Effectiveness \& Evaluation Unit, Royal College of Physicians, London, UK; ${ }^{5}$ Crohn's \& Colitis UK (NACC), Beaumont House, St Albans, UK

Introduction The UK IBD Audit seeks to improve the quality and safety of care for IBD patients throughout the UK by auditing individual patient care and provision and organisation of IBD service resources. The inclusion of an inpatient experience questionnaire in the 3rd round of the audit provided the opportunity to obtain direct patient feedback regarding hospital care.

Methods Teams at participating sites were required to audit a consecutive sample of inpatients with UC and CD. Completion of local clinical data entry for each case triggered the generation of a patient survey comprising a self-completed questionnaire containing items taken from the National Inpatient Survey questionnaire (Picker Institute) with supplementary questions specific to IBD. Aggregated results are presented for all respondents.

Results Response rate: 33\% (data for 2028 adults and 166 children). Results presented as adults [children] where appropriate. Overall care rated as only fair by $7 \%$ [ $6.7 \%$ children] and poor by $2.9 \%[0 \%]$. Overall satisfaction correlated most strongly with rating of how well doctors and nurses worked together. Composite scores across six domains of acute adult care were comparable across all countries (no national differences). Scores for CD were lower than UC in several domains. Scores for consistency and coordination of care and nursing were relatively low compared to general adult inpatients (National Inpatient Survey, 2009). Only $60 \%$ of adults $[76 \%$ children] reported a visit from a specialist nurse. Hospital food: Rated as poor by $20 \%$ [11\%]; "Too little" food provided was reported by $15 \%$ [16\%]; "Never" suited to dietary needs by $15 \%$ [8\%]. No dietician visit in 59\% [26\%]. Pain management: "Ever in pain?" $85 \%$ [83\%]; pain "usually severe" $52 \%$ [52\%]; analgesic medication "not enough" 16\% [12\%]. At least 1 in 10 reported sub-optimal aspects of discharge information such as lack of information about drug side effects, the danger signs to watch for or how to manage their condition after going home.

Conclusion There are many positive findings from the UK IBD inpatient survey-over $3 / 4$ of adults and children rated care as "very good" or "excellent". There are areas for potential improvement. Patients place a strong value on co-ordinated care from experienced staff. Greater provision and involvement of IBD nurses at ward level could play a key role in promoting excellent inpatient care. All admitted IBD patients should receive input from specialist multidisciplinary teams experienced in managing these complex disorders.

Competing interests None declared.

\section{PTU-099 HEALTH-RELATED QUALITY OF LIFE IN GREEK PATIENTS WITH INFLAMMATORY BOWEL DISEASE}

doi:10.1136/gutjnl-2012-302514c.99

K Argyriou,* E Tsakiridou, K Oikonomou, A Kapsoritakis, S Potamianos. Gastroenterology, University Hospital of Thessaly, LARISA, Greece

Introduction Over the last 20 years, health related quality of life (HRQoL) has developed into a scientific index of subjective health status in the management of ulcerative colitis (UC) and Crohn's disease (CD), with the majority of the available data to come from Northern Europe. But limited data are available from Southern Europe. Aim of our study was to characterise the HRQoL in patients with IBD from Central Greece and the investigation of sociodemographic factors and disease characteristics that affect their HRQoL in order to create a bank of data for future comparative studies.

Methods The population of the study consisted of 69 IBD patients who were recruited from the IBD referral centre for Central Greece. Data collection included the usage of the Greek version of IBDQ and a card with the sociodemographic and clinical characteristics of the population. Disease activity was assessed by Harvey-Bradshaw activity index for CD and by Simple Colitis Activity Index for UC. The impact of each characteristic in HRQoL was studied with one and two way ANOVA.

Results $43 \%$ of the patients were suffering from UC and $57 \%$ from $\mathrm{CD}$. The majority were male $(50.7 \%)$ but a prevalence of women was noticed in the CD group. $80 \%$ of patients were $<60 \mathrm{yrs}$. $60 \%$ of 